\title{
Der Zerfall der landschaftlichen Schönheit - kartographisch dargestellt am Beispiel von Grindelwald
}

(Kartenbeilage)

\section{Rechtfertigung eines Versuchs}

Grundsätzlich stimmen wir mit jenen überein, die der Auffassung sind, daß landschaftliche Schönheit letztlich nicht meßbar und quantifizierbar ist. Dazu ist sie eine viel zu komplexe Größe. Sie muß vom Menschen erfühlt und erlebt werden, und jeder Mensch tut das auf seine Weise, nicht immer gleich, je nach seelischer Verfassung, und auch die Landschaft bietet sich je nach Witterung, Jahres- und Tageszeit wieder anders dar. Die Hauptschwierigkeit besteht darin, daß der ästhetische Wert einer Landschaftseinheit nicht nur am Objekt selbst haftet, sondern sich aus der Subjekt-Objekt-Beziehung zwischen Betrachter und Landschaft ergibt.

Seitdem nun aber Landschaft mehr und mehr zu einer «Ressource» geworden ist, die man als «touristisches Potential» vermarkten kann und landschaftliche Werte mehr und mehr zum Gegenstand planerischer und politischer Auseinandersetzung und Bewirtschaftung werden, müssen wir uns mit der Frage befassen. Denn es ist zu vermuten, daß die ästhetische Wertverminderung ein sehr wichtiger Aspekt der landschaftlichen Wertverminderung überhaupt ist und daß ihre Vernachlässigung aus Gründen der Subjektivität und der ungenügenden Quantifizierbarkeit zu Fehlbeurteilungen führen muß. Wahrscheinlich ist die gefühlsmäßig feststellbare Verminderung des ästhetischen Wertes einer Landschaftseinheit ein Signal, das früher auftritt, als meßbare ökologische Schäden festgestellt werden können. Es sollte daher bei den heute so aktuellen Umweltverträglichkeitsprüfungen die ästhetische Wertverminderung auch einbezogen werden.

Wenn wir am Beispiel von Grindelwald einen Versuch der Bewertung und Darstellung der Veränderung der landschaftlichen Schönheit gemacht haben, so ging es dabei nicht darum, exakt-quantitative Methoden auf ein untaugliches Objekt anzuwenden, sondern um die größtmögliche Disziplinierung und Bewußtmachung eines an sich vorwiegend auf intuitiver Schätzung beruhenden Verfahrens. Um in der Auseinandersetzung glaubhaft zu sein, ist es unbedingt nötig, begründbare Kriterien aufzustellen, warum etwas als schön oder als nicht schön und störend empfunden wird. Diese Kriterien können vielleicht nicht allgemein, aber für bestimmte definierbare Personengruppen Gültigkeit haben.
Auch in der Kunst hat man versucht, ästhetische Empfindung rational $\mathrm{zu}$ begründen. Ferdinand Hodler schloß einen Vortrag in Freiburg im Jahre 1897 mit den Worten: «Über allen Werkzeugen des Sehens steht das Gehirn. Es vergleicht die eine Harmonie mit der andern und entdeckt so die wirklichen innern Zusammenhänge der Dinge. Und aus dieser Tätigkeit des Gehirns mit den Erfindungen des Herzens werden neue Herrlichkeiten geboren. Das Kunstwerk wird eine neue Ordnung offenbaren, die den Dingen innewohnt, und das wird sein: die Idee der Einheit.»

Der vorliegende Beitrag fußt auf einer Untersuchung, welche der Verfasser mit 8 Mitarbeitern in den Jahren 1981 bis 1984 begleitend zum MAB-Projekt Grindelwald durchgeführt hat. Das MAB-Programm (Man and Biosphere), international induziert, als nationales Programm durchgeführt, hat den Zweck, Beiträge zum Verständnis der Beziehungen des wirtschaftenden Menschen zu seiner natürlichen Umwelt zu erhalten. Beim Projekt Grindelwald ging es um die Beziehungen zwischen $\mathrm{Na}$ turraum, Berglandwirtschaft und Fremdenverkehr. Beim vorliegenden Beitrag geht es nur um die ästhetischen, das heißt sinnlich, vorwiegend visuell wahrnehmbaren Werte, nicht um ökologische Werte, physiologische Erholungswerte, landwirtschaftliche oder touristische Nutzwerte, wissenschaftliche Forschungswerte und dergleichen, die meist in landschaftlichen Bewertungen berücksichtigt werden.

Das Untersuchungsgebiet war die Gemeinde Grindelwald, ohne das eigentliche Hochgebirge, rund $110 \mathrm{~km}^{2}$ (Abb. 1). Die Resultate und der Beschrieb des Verfahrens sind im MAB-Schlußbericht Nr. 20, 1986 veröffentlicht (GROSJEAN 1986). Die Problematik der landschaftlichen Bewertung und der ästhetischen Wertverminderung wurde auch schon im Sammelband «Umbruch im Berggebiet» dargestellt (GROSJEAN 1984). Auf andere Ansätze landschaftlicher Bewertung kann hier aus Gründen des Umfangs nicht eingegangen werden. Durch einen Beitrag der Stiftung Marchese Francesco Medici del Vascello, Bern, wurde es möglich, die zweite große

Georges Grosjean, Prof. Dr., Geographisches Institut der Universität Bern, Hallerstrasse 12, 3012 Bern. 


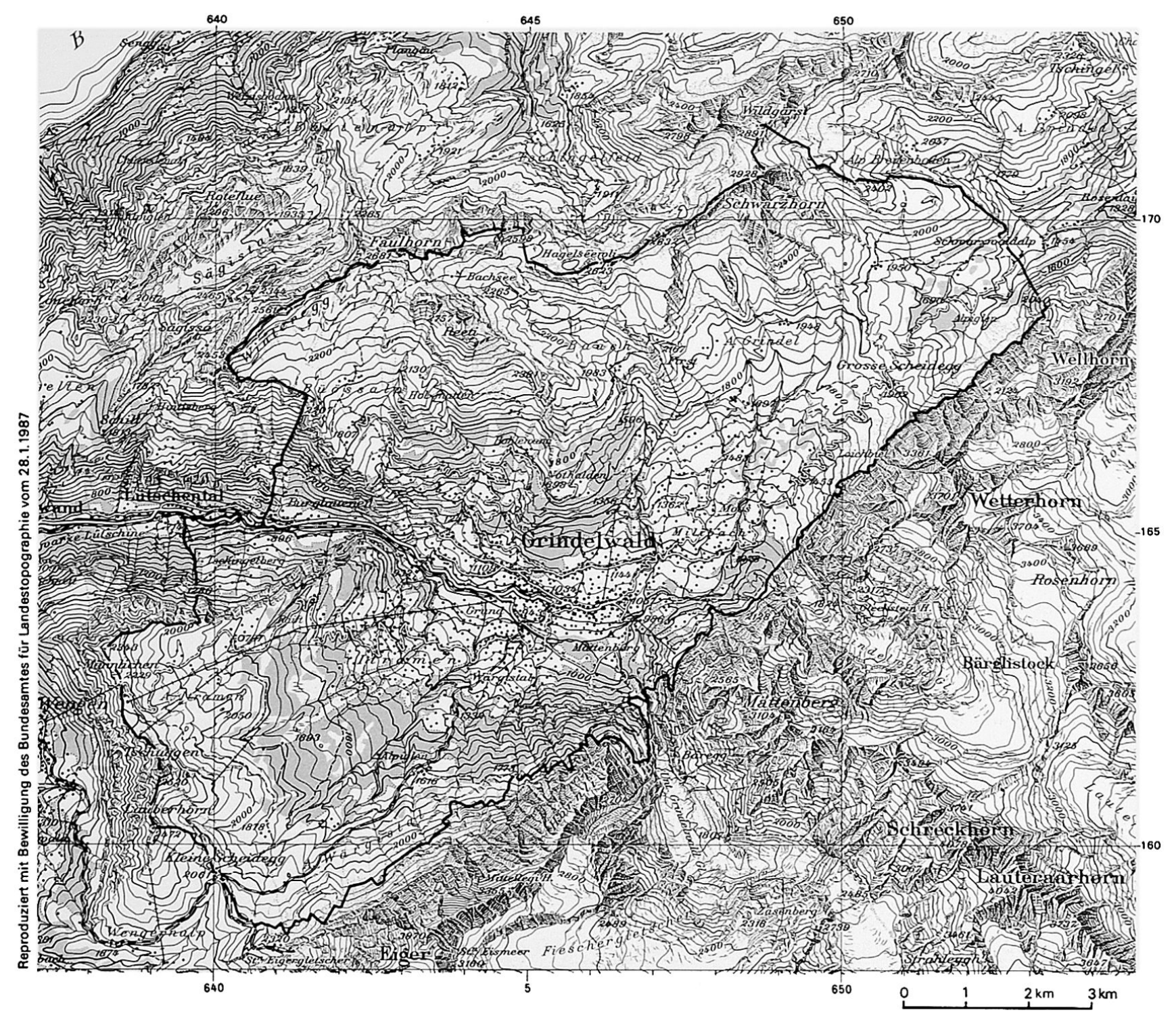

Abb. 1 Das Untersuchungsgebiet

Falzkarte zu reproduzieren und auch diesem Beitrag in den GEOGRAPHICA HELVETICA beizugeben. Der Stiftung sei dafür auch hier herzlich gedankt. Entsprechend dem Inhalt der Karte 2 befaßt sich der vorliegende Beitrag nicht so sehr mit dem Verfahren und den Kriterien der Bewertung, sondern mit der Gewichtung unter dem Aspekt verschiedener denkbarer Personengruppen und mit dem Prozeß der Wertverminderung.

\section{Grundzüge des Verfahrens}

Der ganze Untersuchungsraum wurde in ungefähr gleich große, physiognomisch und in ihren visuellen Beziehungen einigermaßen homogene Einheitsflächen (Bewertungsflächen) eingeteilt. Als zweckmäBige Größe ergab sich empirisch eine Fläche von etwa 0,8 bis $1,5 \mathrm{~km}^{2}$. Für das Untersuchungsgebiet ergaben sich so 93 Bewertungsflächen, zuzüglich 4 kleinere, stärker differenzierte für das Dorfgebiet (Flächeneinteilung auf Karte 2).

Zur Berücksichtigung der Subjektivität wird der Vorgang in zwei Schritte zerlegt: die Bewertung (im engern Sinn) und die Gewichtung.

Die Bewertung soll so objektiv wie möglich sein. Es wird festgehalten, was da ist, wie groß oder wie häufig ein Kriterium in einer Fläche vorhanden ist, wobei man davon ausgeht, da $\beta$ der Beschauer sich in der Fläche bewegt. Für Jahreszeit, Tageszeit und Witterung sind diejenigen Verhältnisse angenommen, in denen der Landschaftscharakter am besten zur Geltung kommt, grundsätzlich aber nicht Winter.

Die Liste für die Bewertung der Einheitsflächen enthält je rund 30 naturräumliche und kulturräumli- 
che Merkmale, die allerdings nie alle in einer Fläche vorhanden sind. Die starke Differenzierung der Merkmalliste soll den Bewerter zwingen, systematisch vorzugehen und genau zu beobachten und abzuwägen. Die Tabellen 1 und 2 enthalten die Gewichtungsfaktoren, sind aber sonst identisch mit den Bewertungsformularen.

Für die Bewertung der Merkmale wird eine einfache Skala von 4 (Maximum) bis 1 (Minimum) verwendet. Keine Angabe bedeutet, da $B$ das Merkmal nicht vorhanden bzw. irrelevant ist. Es werden aber noch keine positiven oder negativen Werte gesetzt. Die Bewertung wird für jedes Merkmal nach Eigenwert und Einflußwert vorgenommen. Der Eigenwert ist das, was in der Fläche selbst vorhanden ist, der Einflußwert das, was von außen auf die Fläche einwirkt (z. B. auch Verkehrslärm) bzw. was man von der Fläche wahrnimmt (Aussicht u. a.). Es scheint uns sehr wichtig zu sein, daß die Einflußwerte berücksichtigt werden. Sie fehlen in vielen Bewertungsansätzen. Die Einflußwerte werden aus naher, mittlerer und großer Distanz beurteilt (GröBenordnung: weniger als $3 \mathrm{~km}$, bis $15 \mathrm{~km}$, mehr als $15 \mathrm{~km})$. Da die Wirksamkeit vieler Merkmale mit der Distanz rasch abnimmt, werden bisweilen bei den Einflußwerten mehrere Merkmale zu einer einzigen Beurteilung zusammengezogen (vgl. Tabellen 1 und 2).

Bei der Beurteilung der Eigen- und Einflußwerte werden nicht nur die sich im Raum befindenden naturräumlichen und kulturräumlichen Objekte beurteilt und summiert, sondern auch deren Strukturwert, der sich aus der Schönheit und Harmonie der Anordnung und Gruppierung der Objekte im Raum ergibt. Eine Beurteilung der landschaftlichen Schönheit muß etwas ganz anderes sein als ein Inventar schützenswerter Natur- oder Kulturobjekte. Überdeckend über die Summierung der Beurteilung von Einzelmerkmalen, und bisweilen auch korrigierend dazu, wird für jede Fläche noch eine Beurteilung verschiedener möglicher Arten psychologischer Erlebniswerte gegeben. Diese Werte fußen auf den sinnlich wahrnehmbaren Raumwerten, sind aber auch von andern Impulsen abhängig, wie z. B. Erinnerungen an historische oder kulturelle Ereignisse. Das Landschaftserlebnis des Vierwaldstätterseegebietes wurde für Generationen von Touristen überhöht durch das Wissen um die urschweizerische Befreiungstradition. Korrigierend wirkt die zusätzliche Berücksichtigung des psychologischen Erlebniswertes «Monotonie» für gewisse Betrachtertypen etwa bei ausgedehnten Wald- oder Heidelandschaften, die sonst wegen der geringen Zahl der Merkmale in der Bewertung schlecht wegkommen würden.

Um der unterschiedlichen Beurteilung durch verschiedene Personengruppen gerecht zu werden, wird jede Bewertung eines Merkmals mit einem subjektspezifischen Gewichtungsfaktor multipli- ziert. Dieser kann nun positiv oder negativ sein, je nachdem die betreffende Personengruppe das Merkmal als positiven Wert oder als Störkomponente empfindet. Die Summe aller positiv und negativ gewichteten Einzelbewertungen ergibt den Gesamtwert einer Einheitsfläche.

\section{Die Gewichtungsprofile}

Da es praktisch unmöglich ist (wenn auch noch viel in dieser Richtung zu erarbeiten wäre), die Bedeutung zu ermitteln, welche zahlreiche Einzelpersonen jedem der einzelnen Merkmale beimessen, haben wir modellhaft drei recht ausgeprägte Gewichtungsprofile erstellt, welche drei Personengruppen charakterisieren, die wir den Naturtyp (N), den Traditionstyp (T) und den Aktivitätstyp (A) nennen. Diese Typen sind zwischen zwei Achsen einzuordnen, deren eine objektbezogen von Natur zu umgewandelter Natur und gebauter Umwelt läuft, die andere subjektbezogen von beharrend, traditionell, retrospektiv, zu modern, vorwärtsorientiert, innovativ.

$A b b .2$ zeigt schematisch die Lage dieserTypen. Die Gewichtungsfaktoren, die wir den einzelnen Merkmalen zuordnen, sind in den Tabellen 1 (Naturtyp) und 2 (Traditionstyp) enthalten.

Der Naturtyp $N$. Wir nehmen an, daß es sich um einen Menschentyp handelt, der sehr naturverbunden und sensibel ist. Er hat auch beträchtliche Kenntnisse in botanischer, zoologischer und ökologischer Hinsicht, so daß er sowohl die großen Linien der Landschaft, Gebirge, Gletscher und Seen, wie auch Kleinformen, und lokale kleine Naturschönheiten würdigen kann, wie kleine Feuchtoder Trockenstandorte, deren Verlust er als große Wertverminderungen empfindet. Bei der Kulturvegetation spricht er noch auf naturnahe Dauerwiesen oder auf traditionelle Obst- oder Rebkulturen an. Es heißt das nicht, daß ein Naturtyp nicht auch etwa auf einen Park, wie auf den Brissagoinseln, anspricht, aber es ist dies nicht typisch und ist bei uns im Sinne der Differenzierung dem TypTzugeordnet. Ebenso nehmen wir im Sinne der Differenzierung der Gewichtsprofile an, da $\beta$ der Naturtyp auch der traditionellen gebauten Umwelt gegenüber indifferent ist. Er hat auch keine entsprechenden Kenntnisse. Es ist auch anzunehmen, daß die Personengruppe, die sowohl auf den Naturraum wie auf den traditionellen Kulturraum gleich sensibilisiert ist, klein sein muß. Jeden modernen baulichen oder technischen Eingriff registriert der Typ $\mathrm{N}$ als empfindliche Störung. Bei den psychologischen Erlebniswerten steht beim Typ N normalerweise "Licht und Weite», das Gipfelerlebnis, obenan. Wir nehmen an, daß derTyp $\mathrm{N}$ Bergsteiger ist, aber nicht Al- 
Tab. 1

Generalis ierungsgrad fur Massstab 1:25'000

\begin{tabular}{|c|c|c|c|c|}
\hline & & Exent & \begin{tabular}{|l} 
Einflhes \\
wert
\end{tabular} & Bemerkungen \\
\hline & & & & \\
\hline 1. & OBERFLÄCHE , RELIEF & & & \\
\hline 1.01 & Mittel- a Grossrelief, Formen & Wa & 10 & \\
\hline 1.02 & Felsmassen quontitativ & 10 & 10 & \\
\hline 1.03 & Kleinrelisf & 10 & 2010 & \\
\hline 1.04 & Schutt, Geroll, Sand & 2 & 211 & \\
\hline 1.05 & Einzelformen, Noturdemb & 5 & $5 \%$ & \\
\hline & & & & \\
\hline 2. & GLETSCHER, GEWA'S & $\overline{S S E R}$ & & \\
\hline 2.01 & Gletscher quantitativ & 10 & $1010{ }^{5}$ & \\
\hline 2.02 & Gletscher qualitotiv & 10 & $1010^{3}$ & \\
\hline 2.03 & Seeflachen & 10 & \begin{tabular}{|l|l|}
10 & 5 \\
\end{tabular} & \\
\hline 2.04 & Seeflöchen - Gliederung & 2 & $5 \sqrt{10}$ & \\
\hline 2.05 & Füsse arantitotiv & 5 & & \\
\hline 2.06 & Flüsse qualitativ & 5 & & \\
\hline 2.07 & Quellen, Bäche & 3 & & \\
\hline 2.08 & Wosserfolle, hydrolog. Noturdenkm & 5 & 31 & \\
\hline & & & & \\
\hline 3. & VEGETATION & & & \\
\hline 3.1 & Wold & & & \\
\hline 3.11 & Node/wold geschlossen & 5 & & \\
\hline 3.12 & Nadeluold offen, in Kamplzase & 10 & & \\
\hline 3.13 & Laubarald geschlassen & 8 & & \\
\hline 3.14 & $\begin{array}{l}\text { Loubwold offen, Boumgrappen } \\
\text { in Kulturland, Wergehils }\end{array}$ & $15 \|$ & 103 & \\
\hline 315 & $\begin{array}{l}\text { Horisontale wo vorikate Gliede. } \\
\text { rumg in geschlassemem Wald }\end{array}$ & 10 & & \\
\hline 3.16 & Pork mit exotischen Arten & 5 & & \\
\hline 3.17 & Eotonisches Noturdenkmal & 5 & & \\
\hline & & & 1 & \\
\hline 3.2 & Offene Naturvegetotio & & & \\
\hline 3.21 & Heide, alpine Rosen, Tundren & 15 & & \\
\hline 3.22 & Feuchtrugetation, Schilf & 20 & & \\
\hline 3.3 & Kulturvegetation & & & \\
\hline 3.31 & Douerwiesen & 10 & 3 & \\
\hline 3.32 & Acker, Futherbou, Gemuise & 1 & & \\
\hline 333 & Reben & 5 & 3 & \\
\hline 334 & Obstboumkulturen & 2 & & \\
\hline 3.35 & Grünflechen, Ziergörten & 2 & & \\
\hline 4 & TIEPUIEIT & & & \\
\hline$\frac{7.01}{4.01}$ & \begin{tabular}{|l} 
Grosswild \\
\end{tabular} & 7 & 7 & \\
\hline 4.02 & Vogel, Kleintiere & 10 & 7 & \\
\hline 4.03 & Schmetterlinge Insekten & 10 & & \\
\hline 4.04 & Fische, Wossertiere & 5 & & \\
\hline & & & & \\
\hline
\end{tabular}

\section{Bearoeiter}

Fel daufnahme
Revision

Datum:
ÄSTHETISCHF LANDSCHAFTSBENERTUNG

LĀNDLICHER RÄUME

Gewichtungsschlüssel N Naturtyp

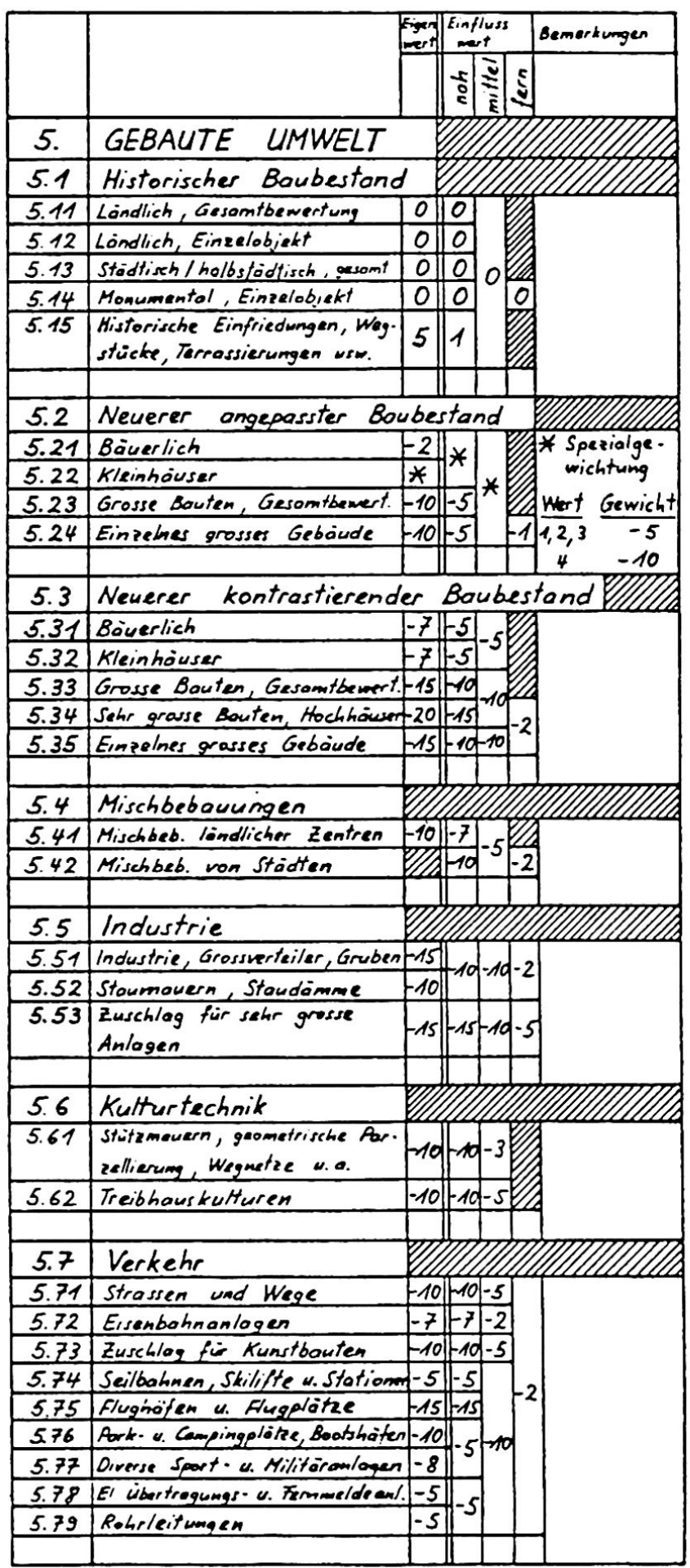

\begin{tabular}{|c|c|c|c|c|}
\hline 6. & PSYCHOLOGISCHER & \multicolumn{3}{|c|}{ ERLEBNISWERT } \\
\hline 6.01 & Romontisch & 10 & Bewertung & \\
\hline 6.03 & Licht und Weite & 20 & & \\
\hline 6.03 & Geboroenheir & 10 & $f E_{\text {gom }}-4$. & \\
\hline 6.04 & Monotonie & 50 & Dimprite & \\
\hline 605 & Kulturall-goistiges Erleben & 0 & & \\
\hline 6.06 & Urbane Ambiance & 0 & & \\
\hline
\end{tabular}


Tab. 2

General is ierungsgrad für Massstab 1:25'000

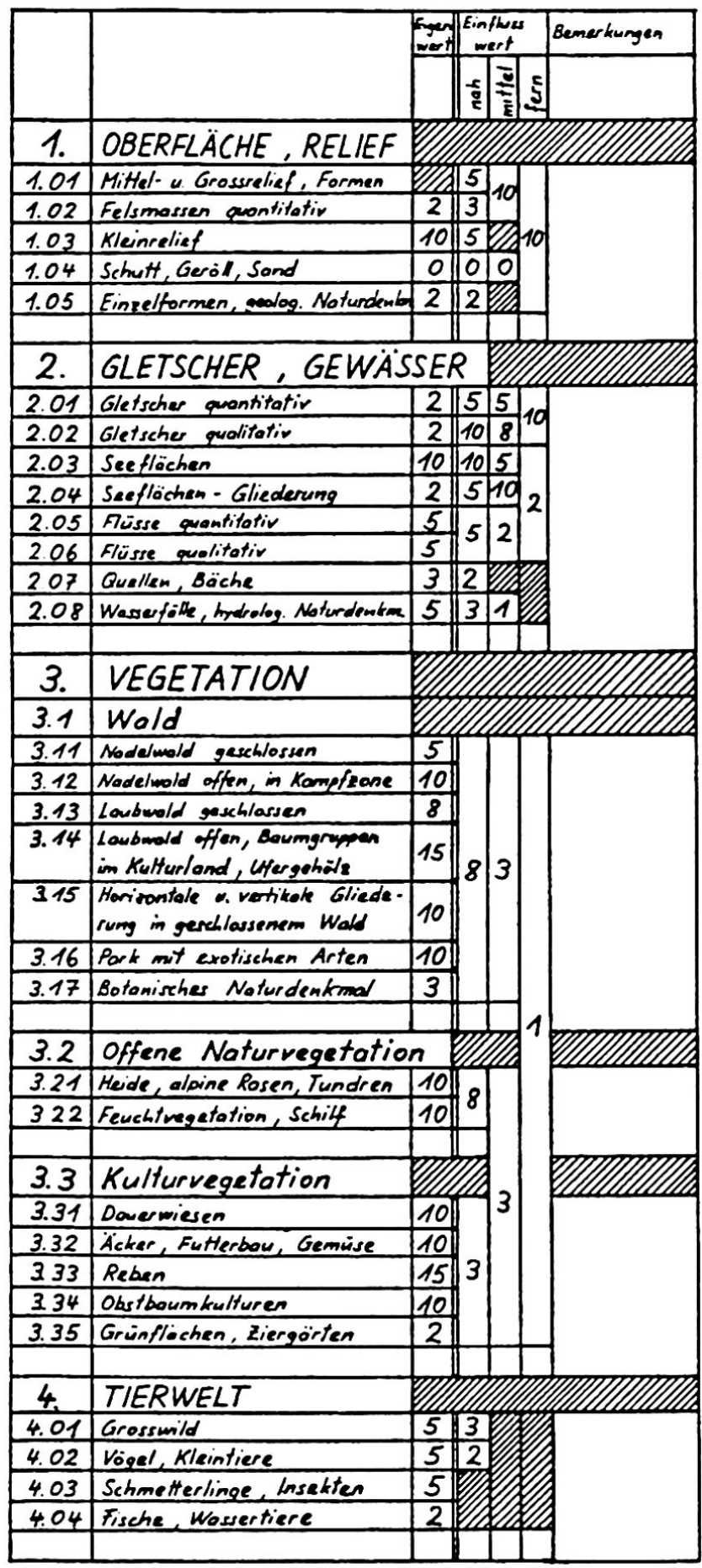

\section{Bearbeiter}

Feldaufnahme:
Revision

Datum:
ÄSTHETISCHE LANDSCHAFTSBENERTUNG

LĀNDLICHER RÄUME

Gewichtungsschlüssel T Traditionstyp

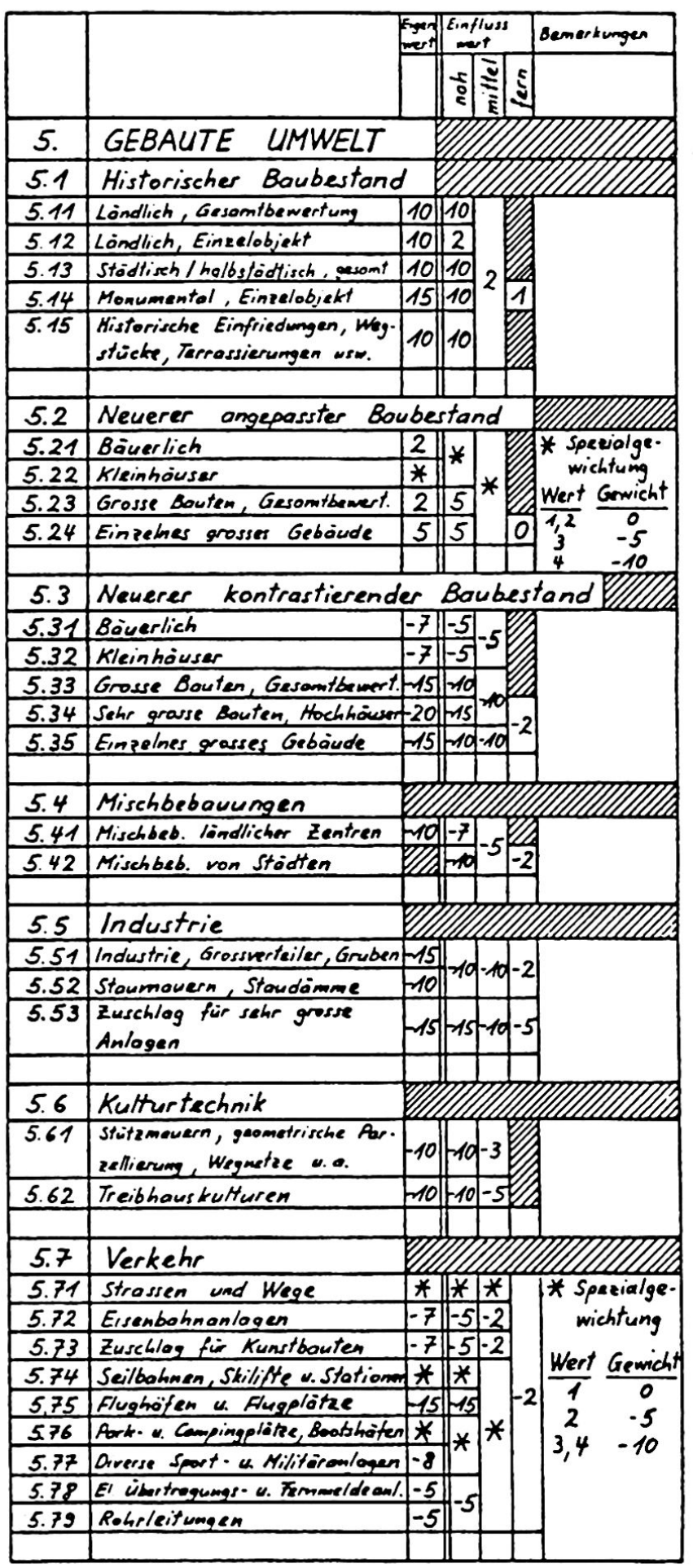

\begin{tabular}{|c|c|c|c|c|}
\hline 6. & PSYCHOLOGISCHER & \multicolumn{3}{|c|}{ ERLEBNISWERT } \\
\hline 6.01 & Romontisch & 10 & Bewertung & \\
\hline 6.02 & Licht und Weite & 15 & & \\
\hline 6.03 & Gebongenheit & 10 & Eym - & \\
\hline 604 & Momotonice & 20 & Eimficas: & \\
\hline 605 & Kullurell - geistiges Erleben & 15 & & \\
\hline 6.06 & Urbane Ambionce & 5 & & \\
\hline
\end{tabular}


pinist oder Kletterer im modernen Sinne. Unter den Naturtypen werden auch jene sein, welche das Einsamkeitserlebnis einer wenig gegliederten Berglandschaft oder eines ausgedehnten Wald- oder Heidegebietes kosten können. Es ist daher bei «Monotonie» (nicht zu verwechseln mit modernen Kultursteppen) ausnahmsweise der außerordentliche Gewichtungsfaktor 50 gesetzt. Das würde bedeuten, daß eine hier mit 4 bewertete Landschaft allein 200 Punkte holen könnte. Dies scheint uns gerechtfertigt in Ansehen, daß solche Landschaften in der Schweiz sehr selten geworden sind und ihr Wert bei der bloßen Summierung der Merkmale nicht in Erscheinung treten würde, abgesehen davon, da $B$ es in der heute mit Häusern und technischen Anlagen vollgespickten Schweiz kaum mehr Räume gibt, welche bei «Monotonie» mehr als den Wert 2 holen könnten. Geringer bewertet ist das romantische $\mathrm{Na}$ turerlebnis, das eher dem 18. und 19. Jahrhundert angehörte, mit wilden, düstern Felsen, wolkenumflorten Gipfeln, kaltblauen Eisströmen, sturmgepeitschten Tannen, den Menschen bedrohend und zur Selbstbehauptung herausfordernd. Ebenfalls wird ein Teil des Typs $\mathrm{N}$ für das Geborgenheitserlebnis zugänglich sein, das in der kleinen, überblickbaren, eher sanften Geländekammer zustande kommt.

Der Traditionstyp T. Hier stellen wir uns einen Typ vor, der ein hohes und differenziertes Verständnis für die historisch gewachsene Kulturlandschaft hat. Er ist auch sensibilisiert für Naturschönheit, aber nicht so differenziert und ausschließlich. Das Hochgebirge von Eis und Fels sieht er lieber aus gewisser Distanz, und eine Landschaft des Mittelgebirges oder des Hügellandes, die reich ist an traditioneller Kulturvegetation, Wald, Gewässern und historischen Baudenkmälern, muß für ihn ebenfalls hohe Punktzahlen erhalten können. Sein Ort liegt daher etwas weiter in Richtung «gebaute Umwelt». Nicht leicht ist die Zuordnung der neueren Bausubstanz zu den Kategorien «angepaßt» oder «kontrastierend» und deren Gewichtung. Wir müssen hier auf die ausführlichen Erörterungen im MAB-Schlußbericht hinweisen (GROSJEAN 1986: 40, 42-49, 124 130, Bildteil 168-177). Grundsätzlich entscheidet nicht der Architekturwert des Gebäudes, sondern dessen strukturelle Einordnung in das Landschaftsund Siedlungsbild. Die von vielen Architekten als unehrliche Nachahmung abgelehnten chalet-typischen Bauten beurteilen wir als umgebungsangepaßt, wenn sie in kleiner Zahl auftreten und sich in die Struktur eines Dorfbildes oder einer historischen Streusiedlung einfügen, dagegen als nicht angepaßt, wenn sie in großer Zahl unstrukturiert auftreten, zu dicht gegenüber der bäuerlichen Streusiedlung, zu locker für ein Dorf, wenn sie starr an geraden Straßenlinien angeordnet sind oder durch ihre Größe als Fremdkörper erscheinen. Immerhin wird man die Verwendung von $\mathrm{Holz}$, die geneigten Dachflächen, allenfalls die Fassadengliederung als mildernde Elemente berücksichtigen. Ein einzelnes großes Gebäude kann, wenn es als alleinige Dominante in einer Siedlung oder durch die Erkennbarkeit eines Ordnungsprinzips einen fruchtbaren Kontrast darstellt, als integriert betrachtet werden (z. B. Kirche, Burg oder Schloß im Dorfbild) und dann entweder dem historischen Baubestand (5.14) oder dem neueren angepaßten Baubestand (5.24) zugeordnet werden. Neue Bauten aber, die regellos und beziehungslos zum historischen Baubestand stehen, werden dem kontrastierenden Baubestand zugeordnet. Aus diesen Überlegungen erklärt sich, warum wir beim Typ T für den neuern angepaßten Baubestand noch geringe positive Gewichtungsfaktoren setzen, beim Typ $\mathrm{N}$ dagegen durchwegs negative, in Ansehen, daß der Typ N die Landschaft am liebsten ohne menschliches Beiwerk sieht. Bei den psychologischen Erlebniswerten nehmen wir bei Typ T etwas geringere Erlebnisfähigkeit für «Licht und Weite» und für «Monotonie» an, dagegen einen hohen Wert für «kulturell-geistiges Erleben» und auch etwas für «urbane Ambiance». Der trotzdem noch hohe Gewichtungsfaktor (15) für «Monotonie» erklärt sich aus der Notwendigkeit der Korrektur der bei monotonen Landschaften nicht zweckmäßigen additiven Methode. Um der Annahme gerecht zu werden, daß bei Typ T die Reizschwelle gegenüber Bauten, Verkehr und andern technischen Anlagen höher ist als beim Typ N, haben wir bei verschiedenen Merkmalgruppen progressive Gewichtungsfaktoren eingesetzt.

Der Aktivitätstyp A. Aus Raumgründen verzichten wir hier auf die Wiedergabe des Gewichtungsprofils in Tabellenform und auch auf einen eingehenderen Beschrieb. Man wird dieses Profil auch ohne viel Erklärung verstehen. Angenommen ist ein Menschentyp, dessen Erleben mehr in der sportlichen Aktivität liegt als im ästhetischen Landschaftserlebnis. Er braucht nicht ein ausgesprochenerWintertyp zu sein. Er kann auch im Sommer als Tennisspieler, Golfspieler, Wassersportler oder Sportkletterer auftreten. Da wir ausdrücklich hier den sportlichen Aktivitätswert eines Raumes nicht beurteilen, ergibt sich, da $B$ wir beim Typ A fast durchwegs geringere Gewichtungsfaktoren einsetzen als bei den beiden andern Typen. Wir nehmen zwar an, da $ß$ auch der Typ A nicht unempfindlich ist für landschaftliche Schönheit. Aber er erfaßt sie nur im Vorbeigehen, in großen Zügen, Gipfelform, Gletscher, See - nicht im intimen Detail, wo man verweilen muß. Auch für historischen Baubestand hat Typ A etwas übrig, aber ohne viel Differenzierung. Alles Übrige empfindet 
er meist nicht als störend (Faktor 0), mit Ausnahme etwa von Industrie und Verkehr, wenn sie im Übermaß auftreten (progressive Gewichtungsfaktoren mit hoher Reizschwelle). Bei den psychologischen Erlebniswerten steht "Licht und Weite» obenan (Sonnen auf der Terasse des Bergrestaurants oder am Seeufer), «Monotonie» kann auch noch etwas bedeuten, und für «urbane Ambiance» (Flanieren in den Ladenstraßen des Dorfes) hat A recht viel übrig. Durch zuviel Verkehr kann diese Ambiance empfindlich geschmälert werden.

In $A b b .2$ ist auch angedeutet, wo die Gäste Grindelwalds in bezug auf diese Gewichtungstypen wirklich liegen. Als vereinfachte Quintessenz aus sehr differenzierten Untersuchungen liegen etwa $60 \%$ der Befragten in einem mittleren Bereich zwischen N, T und $\mathrm{A}$, aber doch deutlicher bei $\mathrm{N}$ und $\mathrm{T}$. Weitere $30 \%$ tendieren näher zu N und T, und nur $10 \%$ gehen stärker gegen A (WIESMANN, 1986 und SCHEURER, 1986, gestützt durch andere Untersuchungen im Rahmen des MAB-Programms: AUCHLIN, VIETTIVIOLI, 1983, KNEUBÜHL, 1984).

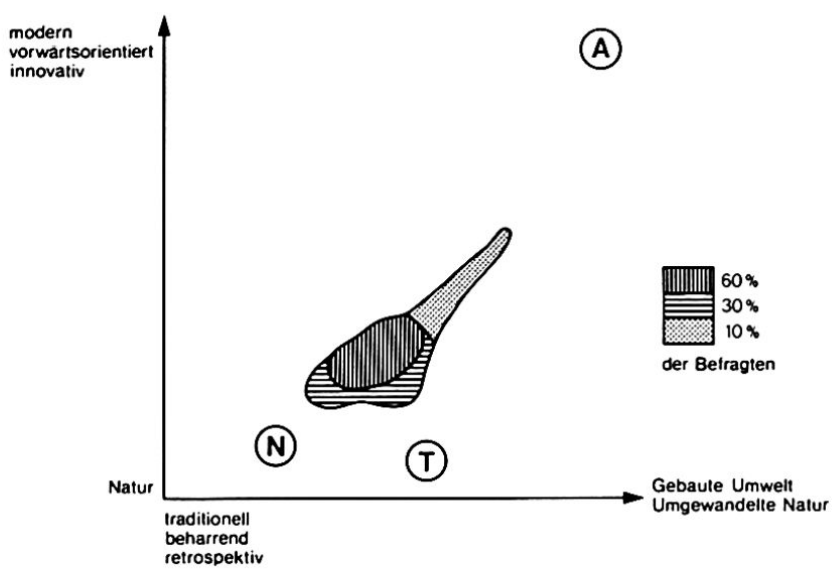

Abb. 2 Die Lage der Gewichtungsprofile N, T und A und die Lage der Gäste von Grindelwald

\section{Die fünf Zustände}

Die Karte 2, die als Falzkarte beigegeben ist, enthält in 15 Teilkarten 1: 100000 die Punktewerte für die drei Gewichtungsprofile in fünf verschiedenen Zuständen, welche den Zerfallsprozeß $B$ der landschaftlichen Schönheit von 1850 bis in die Zukunft veranschaulichen. Der Zustand 1981 beruht auf der Felderhebung des betreffenden Jahres. Der Zustand 1906 entspricht dem Zustand gegen Ende des ersten großen Entwicklungsschubs. Das Jahr entspricht dem Datum der Karte, die dem Werk von
FRIEDLI (1908) beigegeben ist. Dieses Werk war mit seiner für die damalige Zeit guten Bebilderung nebst anderem Bild- und Kartenmaterial die Grundlage für die Rekonstruktion des Zustandes 1906. Der Zustand um 1850 zeigt Grindelwald zur Zeit des letzten Höchststandes der Gletscher und vor dem großen Entwicklungsschub, mutmaßlich das von heute aus denkbare Maximum der landschaftlichen Schönheit Grindelwalds. Die Rekonstruktion erfolgte vorwiegend auf der Basis von Bildmaterial, das für jene Zeit besonders reichlich vorhanden und außerdem bei ZUMBÜHL (1980) umfassend reproduziert ist. Die gegenseitigen Sichtverbindungen zwischen den Flächen wurden anhand des großen Reliefs $1: 10000$ des zentralen Berner Oberlandes von Simon Simon im Schweizerischen Alpinen Museum ermittelt.

Für die zukünftige Entwicklung wurden zwei der Szenarien zugrunde gelegt, welche allgemein für das MAB-Projekt Grindelwald erarbeitet worden sind (WIESMANN. SCHEURER. APEL, in Vorbereitung). Da Stagnations- oder Rezessions-Szenarien im landschaftlichen Aspekt Grindelwalds kaum viel Veränderung gebracht hätten, wurde mit zwei Szenarien gearbeitet, die von einer weitern quantitativen Entwicklung des Fremdenverkehrs ausgehen, entsprechend dem Trend, im Rahmen des gegenwärtigen Bauzonenplans. In beiden Szenarien wird mit einer geringen Zunahme der Einwohnerzahl, aber mit einer Zunahme der Fremdenbetten um 1059 in der Hotellerie und um 4057 in der Parahotellerie gerechnet. Szenario 1 (Konzentration) geht von der Annahme aus, daß der Ausbau vorwiegend in den dorfnahen Zonen südlich und südwestlich des Dorfes im Bereich Grund, Itramen und Wärgistal stattfindet. Es werden vor allem größere Bauten im Stile überdimensionierter Chalets erstellt. Es werden keine neuen touristischen Transportanlagen mehr gebaut, aber dafür die Kapazität der bestehenden verdoppelt. Das Schwergewicht der touristischen Aktivitäten liegt somit weiterhin im Bereich Kleine Scheidegg, Männlichen und First. Im Sinne freier Kombinierbarkeit wird für die Landwirtschaft angenommen, daß derTrend zur Konzentration in größeren Betrieben nachläßt und $\mathrm{da} B$ sich die bestehenden Betriebs- und Siedlungstradition zufolge ausreichender Nebenerwerbsmöglichkeiten erhält, mit gewissen Einbußen im Vorsaßbereich. Allgemein kommt es nicht zu einem Zusammenbruch der landwirtschaftlichen Nutzung in Randlagen. Die gegenwärtige enge Symbiose zwischen Landwirtschaft und Fremdenverkehr bleibt bestehen.

Im Szenario 2 (Ausuferung) wird angenommen, da $\beta$ im ganzen Bereich der heutigen Bauzonen, also auch nordwestlich, nördlich und östlich des Dorfes in lockerer Weise, vorwiegend mit Kleinhäusern im Chaletstil gebaut wird. Im Süden des Dorfes ist eine Umfahrungsstraße gebaut worden, welche den Verkehr von Interlaken unmittelbar zu großen Park- 
plätzen im östlichen Dorfteil führt. Die zur Zeit umstrittene Gondelbahn auf die Große Scheidegg ist gebaut. Das Gebiet beidseits der Großen Scheidegg ist durch mehrere neue Ski- und Sessellifte erschlossen. In der Landwirtschaft geht der Trend nach gröBern, von Zusatzerwerb unabhängigen Betrieben weiter. Es kommt zur Abkopplung von Tourismus und Landwirtschaft; Randlagen werden nicht mehr bewirtschaftet, die traditionelle landwirtschaftliche Streusiedlung wird aufgegeben zugunsten moderner großer Zentralscheunen am Dorfrand und großen, auffälligen Alpställen. Unter dem Einfluß der Intensivierung verarmt die Vegetation; der Vorsaßbereich wird zu Wald.

\section{Der Zerfall der ästhetischen Werte von 1850 bis Szenario 2}

Die absolute Werteskala mit den Kategorien: «auBerordentlich", "sehr schön» und "schön» wurde durch vergleichende Bewertung anderer schweizerischer alpiner und außeralpiner Landschaften gewonnen. Es zeigte sich dabei, daß Flächen mit 400 bis 550 Punkten sehr selten sind. Wenn in Grindelwald 1850 bei Typ $N$ von 97 Bewertungsflächen deren $38 \mathrm{im}$ Bereich «außerordentlich» und weitere 57 im Bereich «sehr schön» liegen, so zeigt dies, daß das Tal von Grindelwald um 1850 - und bedingt auch heute noch - zu den absolut schönsten Landschaften gehört, die es in der Schweiz und im Alpenraum gibt. Die höchsten Werte werden beim Typ N im sehr vielgestaltigen Wald- und Vorsaßgürtel erreicht, in welchem zumeist auch die bedeutendsten ökologischen Werte liegen, der aber ökonomisch am labilsten ist. Ein imposantes Schwergewicht sehr hoch bewerteter Flächen zeigt sich 1850 im Bereich gegen die Große Scheidegg, wo der Eisstrom des Obern Gletschers großartige Kontraste zu den saftiggrünen, mit großen Bäumen bestandenen Matten setzt. Bei Typ Tliegen in allen Zuständen die Werte etwas niedriger als bei $\mathrm{N}$, was zeigt, daß die Schönheit des Tales von Grircúiwald eindeutig bei den naturräumlichen Komponenten liegt. Die höchsten Werte erscheinen fürTyp 'iuñ $1850 \mathrm{im}$ Ostteil des Dorfes, wo mit der Baugruppe der Kirche, mit Pfarrhaus und Pfrundscheune, mit den alten malerischen Holzzäunen und großen Ahornbäumen, mit den südlichen Zypressen auf dem Friedhof, vor dem Hintergrund der Fiescherlücke, von welcher der kaltblaue, serakzerklüftete Untere Gletscher sich weit ins Tal vorschob und aus welcher auch im Winter Sonnenlicht auf diesen Teil des Tales fällt, eine einmalig schöne Verbindung natur- und kulturräumlicher Elemente entstand. Für den Typ A, dem um 1850 auch die englischen Alpinpioniere mit ihrem Leistungsstreben zugehörten, sind die Akzente etwas anders gesetzt. Zwar erscheint auch der Einfluß der beiden Gletscher, sonst aber sind es die hochgelegenen Partien, welche erhöhte Werte zeigen.

Für die Wertverminderung bis 1906 sind folgende Ursachen verantwortlich:

- Der Rückzug der beiden Gletscher. Für alle drei Typen steigen die von den Gletschern beeinflußten Flächen um eine bis mehrere Klassen ab.

- DerVerlust der historischen Bausubstanz im mittleren und westlichen Teil des Dorfes durch den Brand von 1892 und deren Ersatz durch meist stillose, bisweilen sehr große Hotelbauten und halbstädtische Mischbebauung. Zerstörung des Baumbestandes im Dorf. Dieses wirkt kahler als heute. All dies bewirkt eine massive Aushöhlung an Schönheit im Dorfbereich und in den visuell vom Dorf beeinflußten Teilen südlich und südwestlich des Dorfes, in den Auswirkungen spürbar bis auf die Krete Kleine Scheidegg-Männlichen.

- Entstehung neuer Verkehrsanlagen: Postkutschenstraße von Zweilütschinen her, Schmalspurbahnen von Interlaken (1890) und auf die Kleine Scheidegg (1893), Einführung der Straße entlang der Bahnlinie ins Dorf. Die Auswirkungen sind meist auf die direkt betroffenen und anstoßenden Flächen begrenzt.

Anderseits sind 1906 noch der größte Teil der alten Dachbedeckungen (außerhalb des Dorfes) und der alten Einfriedungen intakt. Der Wald ist durch Übernutzung teilweise schlechter als heute. Aufforstungen laufen an. Bemerkenswert ist, $\mathrm{da} \beta$ die ganze Talflanke nördlich und nordöstlich des Dorfes gegen die Alpen Baach, Grindel und Große Scheidegg von den Veränderungen im Dorf nicht betroffen ist, weil der Richtungswechsel der Talachse und die von der Reeti über Nothalten bis an den Dorfrand vorstoßende Schulter den Raum Grindelwald weitgehend in zwei visuell getrennte Kammern einteilt. Dies müßte bei einer künftigen Entwicklung berücksichtigt werden.

Bis 1981 haben vorwiegend die folgenden Entwicklungen zu weiterer ästhetischer Wertverminderung geführt:

- Weiterer Rückzug der Gletscherzungen; sie beeinflussen nur noch sehr wenige Flächen.

- Verdichtung des Dorfgebietes, neue sehr große Baukuben; in der Umgebung des Dorfes große Flächen neuer, strukturloser, halbverdichteter Kleinhaus- und Appartementhaus-Überbauungen.

- Rückbildung der älteren Bausubstanz auch außerhalb des Dorfbereichs, Zerfall von Vorsaßbauten, zunehmende Bedeckung mit Wellblech, neue Zentralscheunen (bisher noch von tragbaren Ausmaßen).

- Verschwinden fast aller Einfriedungen, Eindolen von Bächen, Dränage von Feuchtgebieten, starker Rückgang der Obstbäume im Dorfgebiet. 
- Sehr starke Zunahme der Verkehrsanlagen und des Verkehrs: Ausbau der Straße von Interlaken, Stützmauern, Kunstbauten, starke Zunahme des Zugsverkehrs, Lawinengalerien bei der Wengernalpbahn, Entstehung aller heute bestehenden Seilbahnen, Ski- und Sessellifte, 12 an der Zahl, vorwiegend im Bereich Kleine Scheidegg-Männlichen und First-Oberjoch-Alp Grindel; Bau der Straße auf die Große Scheidegg mit regem Kurswagenverkehr, Entstehung zahlreicher asphaltierter Güter- und Walderschließungsstraßen, Sportanlagen, Tennisplätze, Park- und Campingplätze - oft visuell auf mittlere Distanzen wirksam.

All dies führt nun nicht mehr allein zu einer weitern «Aufhellung» des Dorfgebietes und seiner näher beeinflußten Räume in den Karten für die Typen $\mathrm{N}$ und $T$, sondern zu einer fast durchgängigen, flächigen Verminderung der ästhetischen Werte, allerdings noch etwas weniger im Bereich Nord bis Nordost. Positiv stehen zu Buch einige Aufforstungen oder Waldzuwachs, was - mehr aus einem Zufall sogar bei Fläche 7,03 bei Typ N zu einem Klassensprung nach oben in die höchste Klasse führt. Grund ist, daß diese Fläche nur sehr wenige visuelle Verbindungen nach außen hat, wie auch die «resistenten» Flächen 1,04 (Oberer Itramenwald) und 5,03 (Mulde der Alp Baach). Schon stärker aufgelockert und nicht mehr kompakt erscheint das Bild bei Typ T, während bei Typ A sogar gewisse Wertzunahmen im Bereich der Kreten der Kleinen und Großen Scheidegg erscheinen, bedingt dadurch, $\mathrm{da} ß$ wir annehmen, daß der Typ A in der ErschlieBung schöner Naturräume und Aussichtspunkte eine ästhetische Wertvermehrung sehe.

Unter den beiden Szenarien erscheint 1 als eindeutig günstiger. Bei Typ $\mathrm{N}$ ist das helle Loch, das im Dorfgebiet und seinen Nachbarflächen weiter aufgerissen wird, kleiner. Auf der Seite Nord-Nordost ist noch ein großer, mehr als die Hälfte des Untersuchungsgebietes ausmachender, fast kompakter Raum, der mit kleinen Ausnahmen ausschließlich Flächen der Kategorien «außerordentlich» und «sehr schön» enthält. In Szenario 2 sind zwar hier auch noch die meisten Flächen in der Kategorie «sehr schön», aber fast alle sind um eine bis zwei Klassen abgestiegen, «außerordentliche» Flächen gibt es nur noch sehr wenige. Da wir beim TypTeine gegenüber $\mathrm{N}$ weniger differenzierte und weniger intensive Erlebnisfähigkeit für Natur angenommen haben, stehen bei diesem Typ die negativen Punkte verhältnismäßig stärker zu Buche, so daß wir allgemein wesentlich geringere Werte erhalten als fürTyp $\mathrm{N}$, dem trotz aller Störfaktoren die Natur von Grindelwald immer noch sehr viel bietet. In Szenario 2 fällt fürTypTnicht nur die touristische Erschließung des Raumes beidseitig der Großen Scheidegg ins Gewicht, sondern auch noch der Zerfall der traditionellen ländlichen Bausubstanz, so daß für den
Typ $\mathrm{T}$ in Szenario 2 keine einzige Fläche $\mathrm{mehr}$ im Bereich «außerordentlich» liegt. Für den Typ A zeigen beide Szenarien ein eher diffuses Bild ohne großeVeränderung gegenüber 1981, wobei die höheren Werte in den Bereichen Kleine ScheideggMännlichen und First-Große Scheidegg liegen.

\section{Die Diagramme der Veränderung}

Die Karte 2 gibt keine Auskunft darüber, aus welchen Komponenten sich die Punktwerte der einzelnen Bewertungsflächen zusammensetzen, wie groß die positiven und die negativen Punktzahlen und wie groß der Anteil des Eigenwertes und der Einflußwerte sind; ebenso sind weder die absoluten noch die relativen Mutationsbeträge unmittelbar ablesbar. Wir haben daher zur Ergänzung Diagramme der Veränderung entworfen, allerdings nicht für alle 97 Flächen - das hätte mit je drei Gewichtungsprofilen zur völligen Unübersichtlichkeit geführt - sondern nach 11 Teilräumen, die nach Kriterien der Lage, der bisherigen Entwicklungsdynamik und nach der künftigen Prozeßerwartung abgegrenzt wurden. Diese Teilräume sind $(A b b .3)$ :

1. Das engere Dorfgebiet. 2. und 3. Peripherie SW und E, d. h. das weitere Dorfgebiet, Zersiedlungszone, teils in Bauzone, teils noch landwirtschaftlich genutzt. 4., 5. und 6. Talräume SW, NW und $\mathrm{E}$, außerhalb der Bauzone, Dauersiedlungsbereich der Landwirtschaft. 7. Burglauenen. 8. Die Hangfußzone am Eiger. Schließlich der Wald-, Vorsaß- und Alpbereich, 9. Itramen-Wärgistal, 10. Büößalp, 11. Baach-Grindel-Scheidegg.

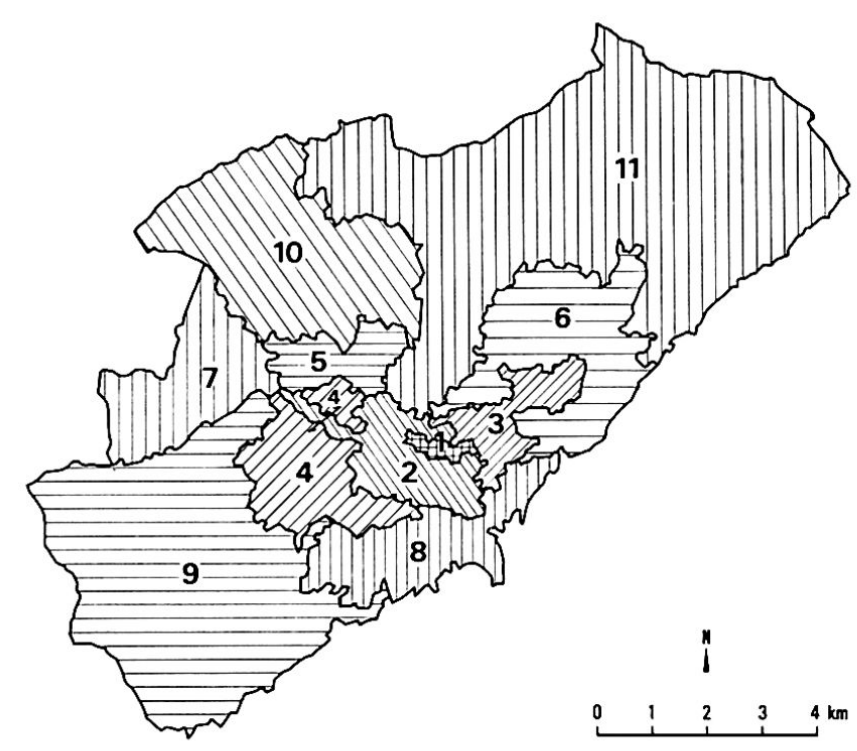

Abb. 3 Teilräume verschiedener Entwicklungsdynamik und Prozeßerwartung. 


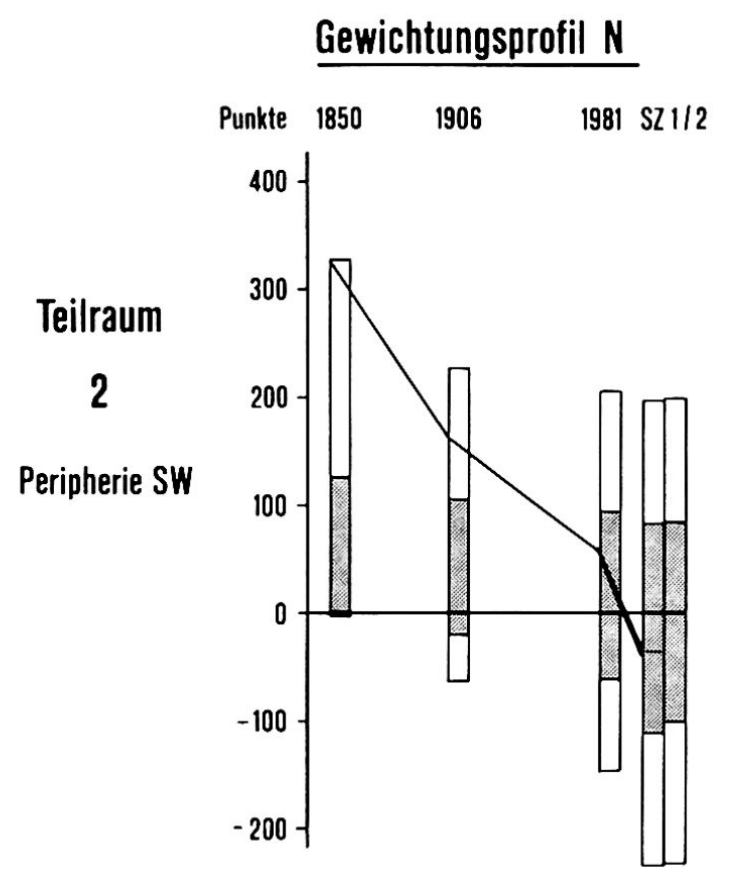

\section{Gewichtungsprofil T}

$1850 \quad 1906 \quad 1981 \mathrm{SZ} 1 / 2$
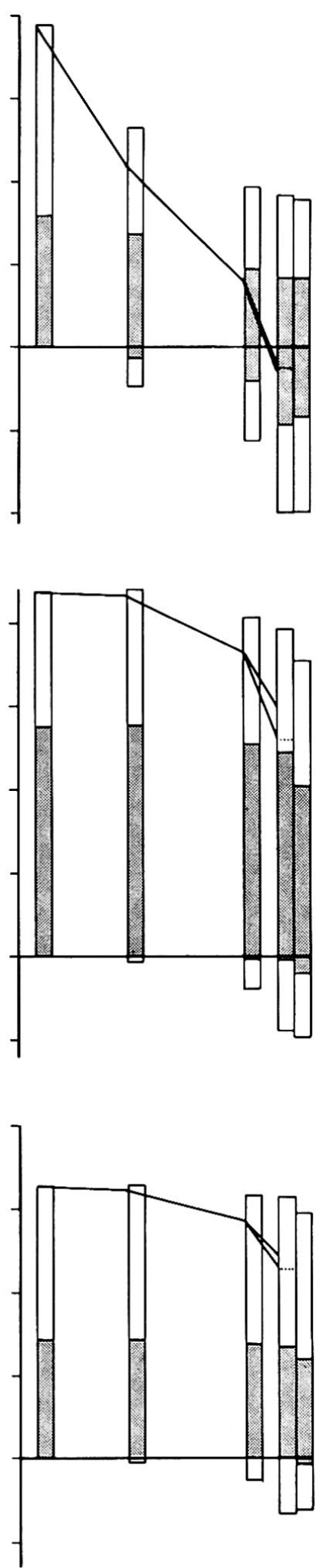

Gewichtungsprofil A

$1850 \quad 1906 \quad 1981 \quad S 21 / 2$
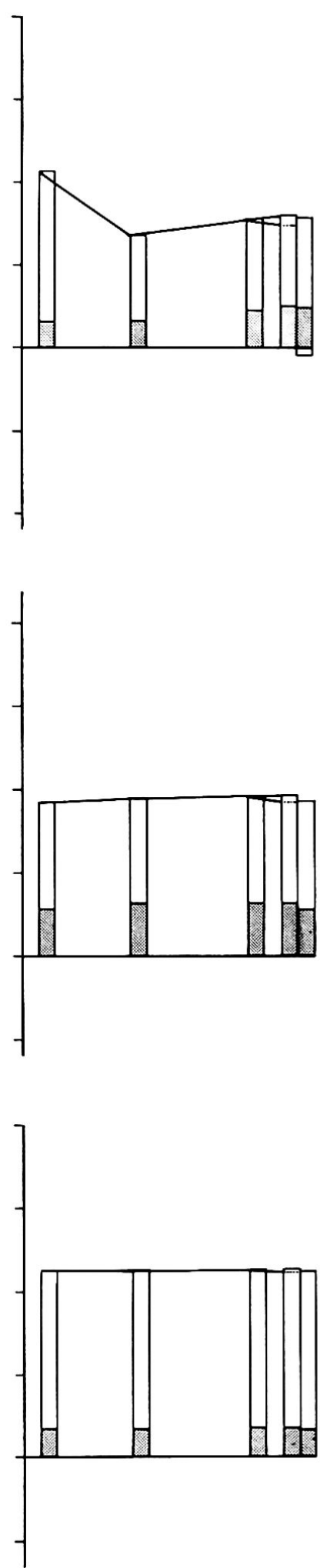

Abb. 4 Diagramme der Veränderung für drei Teilräume und drei Gewichtungsprofile. 
Wir geben in $A b b .4$ drei dieser typischen Diagramme je für die drei Gewichtungsprofile. Die Werte der Teilräume sind als arithmetisches Mittel aus den im Teilraum enthaltenen Flächen ermittelt. Die Stabhöhe über der Nullinie gibt die positive, diejenige unter der Nullinie die negative Punktzahl. Die Lage der Kurve im Stab zeigt den Gesamtwert. Bei den Stäben der Szenarien 1 und 2 ist die Szenario 2 zukommende Kurve mit dem Stab durch eine punktierte horizontale Linie verbunden. Die Eigenwerte sind in den Stäben gerastert, die Einflußwerte ohne Raster. Teilraum 2 ist ein schon durch die bisherige Entwicklung stark betroffener Raum, der in beiden Zukunftsszenarien in die negativen Werte sinkt, wobei die Einflußwerte der nahen Umgebung bedeutenden Anteil haben. Teilraum 5 ist ein vom Dorf entfernterer, noch sehr gut erhaltener bäuerlicher Streusiedlungsraum. Er hat daher bei Typ T einen höhern Gesamtwert. Er ist bereits jetzt durch Einflußwerte beeinträchtigt (Blick auf die wirrsten Verkehrs- und Dorfgebiete) und würde dies noch mehr, vor allem bei Szenario 2. Der Teilraum 10 ist als Alpgebiet mit geringer Erschließung (Sträßchen) der Verminderung des Eigenwertes weitgehend entzogen. Doch wird der Blick ins Tal vermehrt negative Einflußwerte bringen, Szenario 2 zusätzlich Wertverminderungen im Baubestand (Alphütten).

Das MAB-Programm verlangt Aufzeigen von Belastbarkeitsgrenzen. Die landschaftliche Schönheit ist aber kein System, das durch sein «Kippen» andeuten kann, daß die Belastbarkeitsgrenze überschritten wird. Landschaftliche Schönheit ist ein Potential, das einmal auf einem Maximum ist und dann ständig vermindert werden kann. Beschleunigt sich diese Verminderung, dann ist zum mindesten ein Alarmsignal gesetzt. Die Annahme einer Grenze ist in das Ermessen des Menschen gestellt und damit letztlich das Ergebnis eines politischen Entscheides. Die grafische und kartografische Sichtbarmachung des Zerfallsprozesses und seiner Ausmaße kann den Entscheidungsträgern Entscheidungsgrundlagen liefern. Es kann ja auch sein wenn auch nicht beweisbar - daß Verlust der Schönheit sich psychologisch im Menschen niederschlägt und zu Aggression und politischer Unruhe führt.

Viel Potential an Schönheit ist kein Grund, damit sorglos umzugehen; denn in einem Fall wie Grindelwald liegt das Einzigartige gerade in dem sehr hohen Potential. Wenig Potential ist aber auch kein Grund, diesen letzten Rest noch zu zerstören. Einseitige Ausrichtung auf das harmlose Profil A wäre auch ungut, angesichts der Hinweise, da $\beta$ ein solches extremes Profil bei Gästen und Einheimischen kaum vorhanden ist, sondern doch eine Sensibilisierung in Richtung $\mathrm{N}$ und Tfeststellbar ist.

\section{Literatur}

AUCHLIN, P., VIEITI-VIOLI, Ph. (1983): Perception du Pays d'Enhaut par ses touristes (1980-1981). Schlußberichte zum Schweizerischen MAB-Programm Nr. 3, 1983. Rapport final du projet 4.177 , Bern.

BAUMGARTNER, R. (1984): Die visuelle Landschaft, Kartierung der Ressource Landschaft in den Colorado Rocky Mountains (USA). Geographica Bernensia, herausg. vom Geographischen Institut der Universität Bern, Reihe G, Nr. 22, Bern.

BUGMANN, E. (1975): Die formale Umweltqualität. Ein quantitativer Ansatz auf geographisch-ökologischer Grundlage, Solothurn.

BÜRGIN, M., BUGMANN, E., WIDMER, F. (1985): Untersuchungen zur Verbesserung von Landschaftsbewertungsmethoden. Publikationen der Forschungsstelle für Wirtschaftsgeographie und Raumplanung an der Hochschule St. Gallen, Nr. 9, St. Gallen.

FRIEDLI, E. (1908): Bärndütsch als Spiegel bernischen Volkstums. Zweiter Band: Grindelwald. Mit einer Karte über das ständig bewohnte Grindelwald von G. Strasser, Pfarrer, 1906. Bern, 1908, Neudruck 1980.

GROSJEAN, G. (1984): Visuell-ästhetische Veränderungen der Landschaft. In: Brugger A. E., Furrer, G., Messerli, B. und Messerli P.: Umbruch im Berggebiet - Les régions des montagnes en mutation, S. 105-138, 1984, Bern.

GROSJEAN, G. (1986): Ästhetische Bewertung ländlicher Räume, am Beispiel von Grindelwald, im Vergleich mit andern schweizerischen Räumen und in zeitlicher Veränderung. Schlußberichte zum Schweizerischen MAB-Programm Nr. 20, Bern.

KNEUBÜHL, U. (1984): Umweltqualität der Tourismusorte im Urteil der Schweizerbevölkerung. Auswertung einer Befragung im Rahmen der Grundlagen für das Schweizerische Tourismuskonzept. Geographisches Institut der Universität Bern.

MEYRAT-SCHLEE, E. (1983): Werte und Verhalten, Bedeutung und Wirkungsweise von Wertsystemen im Entwicklungsprozeß einer Berggemeinde, aufgestellt am Beispiel von Grindelwald. Schlußberichte zum Schweizerischen MAB-Programm, Nr. 2, Bern.

SCHEURER, T. (1986): Landschaftsbewertung - eine Bewertung der Seele? Ein Beitrag zur Frage nach den Schwellen der Belastung einer Landschaft (erläutert am Beispiel des MAB-Testgebietes Grindelwald) In: Der Mensch in der Landschaft. Festschrift für Georges Grosjean, herausg. von Klaus Aerni, Georg Budmiger, Hans-Rudolf Egli und Elisabeth Roques. Jahrbuch der Geographischen Gesellschaft Bern, Band 55/1983-1985, Bern.

WIESMANN, U. (1986): Landschaftliche Schönheit und touristische Nachfrage. Ergebnisse einer Gästebefragung im MAB-Testgebiet Grindelwald. In: Der Mensch in der Landschaft. Festschrift für Georges Grosjean, herausg. von Klaus Aerni, Georg Budmiger, Hans-Rudolf Egli und Elisabeth Roques. Jahrbuch der Geographischen Gesellschaft Bern, Band 55/1983-1985, Bern.

WIESMANN, U., SCHEURER, T., APEL, H. (in Vorbereitung): Grindelwald im Umbruch. Synthesebericht zum NFP MAB, Testgebiet Grindelwald. Bern.

ZUMBÜHL, H. J. (1980): Die Schwankungen der Grindelwaldgletscher in den historischen Bild- und Schriftquellen des 12. bis 19. Jahrhunderts. Denkschriften der Schweizerischen Naturforschenden Gesellschaft, Band XCII. BaselBoston-Stuttgart. 